\title{
Parasite community interactions: Trypanosoma cruzi and intestinal helminths infecting wild golden lion tamarins Leontopithecus rosalia and golden-headed lion tamarins L. chrysomelas (Callitrichidae, L., 1766)
}

\author{
Rafael V. Monteiro • James M. Dietz • Becky Raboy • \\ Benjamin Beck • Kristel De Vleeschouwer • \\ Andrew Baker • Andréia Martins • Ana Maria Jansen
}

Published online: 13 September 2007

(C) Springer-Verlag 2007

The name of one of the authors got misspelled in the original publication. The correct list of authors must read:

Rafael V. Monteiro, James M. Dietz, Becky Raboy, Benjamin Beck, Kristel De Vleeschouwer, Andrew Baker, Andréia Martins and Ana Maria Jansen

The online version of the original article can be found at http://dx.doi. org/10.1007/s00436-007-0652-2.

\section{R. V. Monteiro · A. M. Jansen}

Lab. Biologia de Tripanosomatídeos,

Instituto Oswaldo Cruz, FIOCRUZ,

Av. Brasil 4365,

Rio de Janeiro, RJ 21040-360, Brazil

\section{J. M. Dietz}

Department of Biology, University of Maryland,

College Park, MD 20742, USA

\section{B. Raboy}

Species Conservation Center,

Smithsonian National Zoological Park,

3001 Connecticut Ave NW,

Washington, DC 20008, USA

B. Beck

Great Ape Trust of Iowa,

4200 SE 44th Avenue,

Des Moines, IA 50320, USA
K. De Vleeschouwer

Center for Research and Conservation,

Universiteit Antwerpen,

Koningin Astridplein 26,

B-2018, Antwerp, Belgium

A. Baker

Philadelphia Zoological Gardens,

3400 West Girard Avenue,

Philadelphia, PA 19104-1196, USA

A. Martins

Associação Mico Leão Dourado,

P.O. Box 109968, Casimiro de Abreu, RJ 28960-970, Brazil

R. V. Monteiro ( $\square)$

Lab. Biologia de Tripanosomatídeos,

Pavilhão Carlos Chagas, $3^{\circ}$ andar, Av. Brasil, 4365,

Rio de Janeiro, RJ 21040-360, Brazil

e-mail: rafael.monteiro@infolink.com.br 